\title{
ACCPM with a nonlinear constraint and an active set strategy to solve nonlinear multicommodity flow problems: a corrigendum
}

\author{
F. Babonneau • J.-P. Vial
}

Received: 7 September 2007 / Accepted: 7 September 2007 / Published online: 30 October 2007

(C) Springer-Verlag 2007

\section{Erratum to: Math Program Ser. B DOI 10.1007/s10107-007-0151-3}

This note points out errors in three instances of the data test set used in the paper [1] appearing in this issue. We used ACCPM to obtain a solution with relative accuracy $10^{-5}$ with the new data sets. We observed similar behavior of the algorithm. We also indicate how to download the data we used initially.

\section{The corrigendum}

Shortly before the publication of [1] in this issue, our attention has been drawn on errors on the data sets we used for three problems, namely: NDO22, Barcelona and Philadelphia. In NDO22 we misprinted the capacity on the arc between node 14 and node 8: a 3 became 13. In Barcelona and Philadelphia, we did not use the correct parameters for the BPR function (the results for the Kleinrock function are not affected). At the time we completed the research, we were not aware of published results on the optimal values for Barcelona and Philadelphia, which did not make it possible to detect the error.

The purpose of this note is twofold. We first show the behavior of ACCPM for solving the three problems with the correct data with a relative accuracy $10^{-5}$. Second, we

The online version of the original article can be found under doi:10.1007/s10107-007-0151-3.

\footnotetext{
F. Babonneau · J.-P. Vial $(\varangle)$

ORDECSYS, Geneva, Switzerland

e-mail: jpvial@ordecsys.com

F. Babonneau

e-mail: fbabonneau@gmail.com
} 
Table 1 New experiments using a cutting surface and column elimination

\begin{tabular}{lllrrrrr}
\hline Problem ID & Objective & $z^{*}$ & Nb cuts & Outer & Inner & CPU & $\%$ Or \\
\hline ndo22 & Kleinrock & 103.412 & 12 & 12 & 74 & 0.2 & 8 \\
ndo22 & BPR & $1.86767 \times 10^{6}$ & 6 & 6 & 32 & 0.1 & 0 \\
Barcelona & BPR & $1.22856 \times 10^{6}$ & 32 & 50 & 189 & 7.8 & 35 \\
Philadelphia & BPR & $2.24926 \times 10^{8}$ & 158 & 374 & 1,595 & $8,406.1$ & 75 \\
\hline
\end{tabular}

The relative accuracy is $10^{-5}$

give the address [2] where to download the network data we used in the experiments in [1].

In the new experiments reported in Table 1, the settings of ACCPM are the same as those which led to the results of Table 5 (for NDO22) and Table 6 (for the three problems) in [1]. We also give the correct optimal objective values. The results show that ACCPM solves NDO22 and Barcelona in a comparable number of iterations while Philadelphia, which has a significantly higher congestion, needs more iterations.

Acknowldegments We are indebted to K. Kiwiel who pointed out the error for the three problems.

\section{References}

1. Babonneau, F., Vial, J.-P.: ACCPM with a nonlinear constraint and an active set strategy to solve nonlinear multicommodity flow problems. Math. Prog. (this issue)

2. Babonneau, F.: Alternate data sets for the multicommodity flow problems Barcelona and Philadelphia. http://www.ordecsys.com/oboe/AlternateData.tar.gz 